\title{
«Auch bei mir geht es um den Menschen»
}

\section{Daniel Lüthi}

Text und Bilder

danielluethi(at)gmx.ch
Spiez am Thunersee: Wir blicken auf die Blüemlisalp, das Stockhorn und die majestätische Pyramide des Niesen gleich gegenüber. Wir blicken nach vorn, auf den letzten Tag dieses Monats, den letzten offiziellen Arbeitstag von Heiner Frost. Und wir blicken, wie er es nennt, zurück auf «ein schillerndes medizinisches Leben». 50 Jahre erlebte Medizingeschichte - nicht in der Arztpraxis oder im Spital vor allem, sondern im Labor und im Büro, in der Forschung, Wissenschaft und Lehre.

\section{Arbeit im Spannungsfeld}

Heiner Frost spricht mit Bedacht, wählt seine Worte sorgsam aus, wägt ab. Die Gewissenhaftigkeit, ja Akribie findet sich bei ihm auch in der Sprache. Und auch hier legt er Wert auf die Verständlichkeit. «Mit dem Begriff «Clinical Reviewer〉 bin ich nicht glücklich», bemerkt er, «in keiner Landessprache der Schweiz wird er dem gerecht, was wir machen.» Das übrigens sagt einer, der mehrheitlich nicht bloss englisch gearbeitet hat, sondern vielfach immer noch englisch denkt. Also: Was macht ein Clinical Reviewer? «Wer auf wissenschaftlichem Gebiet eine Review schreibt, vermittelt eine Übersicht über das ganze Wissensgebiet.» Der Satz sitzt, Frost lehnt sich zurück, schaut in die Ferne. "Früher, als Swissmedic noch IKS hiess, Interkantonale Kontrollstelle für Heilmittel, nannte man uns «klinische Assessoren». Wir arbeiten im Bereich Zulassung, wir prüfen Gesuche für neue Arzneimittel, oder für neue Indikationen von bereits zugelassenen Medikamenten. Wir Reviewer lesen und beurteilen den klinischen Teil eines Zulassungs-

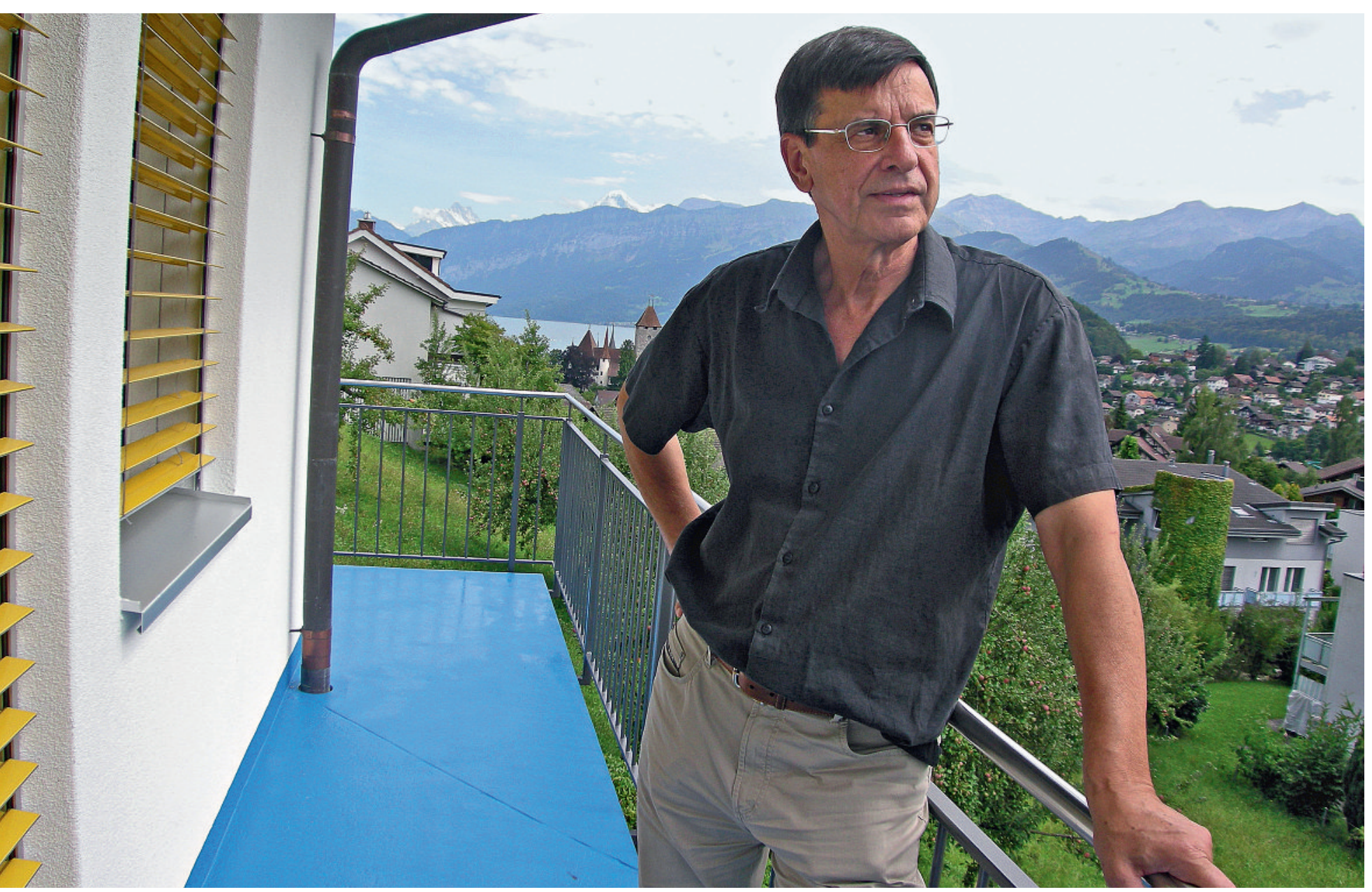


dossiers, also dessen grössten Teil.» «Wir», sagt Frost immer wieder, nicht «ich» - allein dies deutet darauf hin, dass er sich nicht als Einzelkämpfer sieht, auch wenn die Beschreibung seiner Arbeit oft an die Einsamkeit eines Langstreckenläufers denken lässt.

Hunderte von Bundesordnern werden da jeweils angeliefert, «oft 50000 bis 100000 Seiten voller Zahlen, Statistiken, Tabellen und Grafiken». Zunehmend kommen die Gesuche nur noch auf elektronischem Weg ins Haus. In Zellkulturen und Tierversuchen, später auch in klinischen Studien am Menschen ist das Arzneimittel, um das es geht, vorher getestet worden. Die Datensätze, die im Büro von Heiner Frost landen, sind die Ergebnisse klinischer Studien. Seine Spezialität: biotechnologisch hergestellte Stoffe wie Interferone, monoklonale Antikörper und andere Proteine, und damit Medikamente gegen entzündliche, allergische, rheumatische, onkologische oder in letzter Zeit auch ophthalmologische Erkrankungen.

Ein Clinical Reviewer arbeitet in einem Spannungsfeld - Interessen von Industrie, Patienten und Ärzten treffen hier aufeinander. Zum Zeitdruck 20 Arbeitstage stehen Frost für die erste Phase eines Gutachtens zur Verfügung - kommt der Druck von Öffentlichkeit und Politik.

\section{Nutzen und Risiken}

Für die betroffenen Firmen geht es um viel Geld, «die wollen die aufgelaufenen Investitionen, Milliarden zum Teil, im Markt natürlich möglichst schnell zurückbekommen», sagt Heiner Frost. Er und seine Kolleginnen und Kollegen kümmern sich nicht um wirtschaftliche Aspekte, weder um Preise noch um Gewinne, weder um Ausgaben noch um Einnahmen. Pharmakokinetik und Pharmakodynamik, erwünschte und unerwünschte Wirkungen, Haupt- und Nebenwirkungen, Wirksamkeit und Sicherheit: Das sind ihre Themen. Ein Clinical Reviewer wägt immer wieder Nutzen und Risiken sorgfältig gegeneinander ab. Heiner Frost drückt es so aus: «Wir sind medizinische Gutachter von Heilmitteln. Jedes Mal wollen wir ein unabhängiges, neutrales Gutachten erstellen, das nachvollziehbar, transparent und kohärent ist.» Verständlich eben, auch wenn der Bescheid letztlich negativ sein kann, ein Gesuch also abgelehnt werden muss, was immer wieder geschieht.

Frost selber konnte seinen eigenen Anspruch offensichtlich immer erfüllen: «In meinen 17 Jahren als Reviewer hatte ich keinen einzigen Rekurs. Ja: Dies ist ein Erfolgserlebnis für mich.» Hat er in seiner Gutachter-Tätigkeit direkte Druckversuche erlebt? «Die haben sich erstaunlich in Grenzen gehalten.»

Wie auch immer: Auch vor dem Computer spürt ein Mediziner die Last der Verantwortung.

\section{Autonomie und Souveränität}

Fühlt sich ein Arzt, der Daten sieht statt Patienten, noch als Arzt? «Auch bei mir geht es um den Menschen», antwortet Frost. «Ich habe halt nur indirekt mit ihm zu tun. Hier aber zeigt sich die ganze Kom-

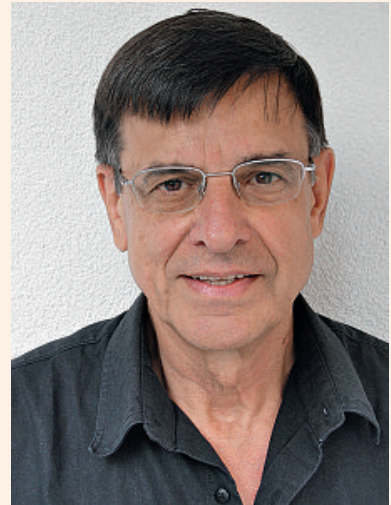

\section{Heiner Frost}

Dr. med. Heiner Frost wurde 1942 in Schlesien geboren und wuchs ab 1945 in Bonn auf.

Er studierte Medizin in Bonn, Freiburg und Heidelberg, wo er sein Studium 1967 abschloss. Dann war er Assistenzarzt in Innerer Medizin an den Universitätskliniken in Bonn und Zürich und Mitglied des Instituts für Immunologie in Basel. Von 1976 bis 1980 arbeitete er an der Wayne State University in Detroit (USA) als Assistant Professor an den Departementen Innere Medizin und Immunologie/Mikrobiologie, wo er seit 1984 auch Adjunct Associate Professor ist. Zwischen 1981 und 1994 bekleidete er verschiedene Funktionen in der klinischen Forschung bei Ciba-Geigy in Basel und Summit/ New Jersey, vor allem in den Bereichen Allergologie, Immunologie, Infektiologie und Onkologie. 2007 bis 2009 war er bei der Weltgesundheitsorganisation WHO in Genf Vorsitzender eines klinischen Entwicklungsteams für afrikanische Trypanosomiasis (Schlafkrankheit).

Seit 1995 ist Heiner Frost Clinical Reviewer (medizinischer Gutachter) bei Swissmedic, dem Schweizerischen Heilmittelinstitut in Bern. Hier beurteilt er die Wirksamkeit und Sicherheit von biotechnologisch hergestellten Heilmitteln. Dazu gehörten in den letzten 10 Jahren Interferone, monoklonale Antikörper und andere Proteine.

2005 schloss er an der Universität Zürich ein Masterstudium in angewandter Ethik ab.

Heiner Frost ist Vater von zwei Kindern, und er hat drei Enkelkinder. Er lebt zusammen mit seiner Frau in Spiez am Thunersee.

plexität klinischer Daten: Ich muss sie bis zum einzelnen Patienten zurückverfolgen können. So interessiert mich: Gab es ein unerwartetes Ereignis? Einen Autounfall oder eine zusätzliche Erkrankung zum Beispiel? Eine klare Nebenwirkung des neuen Arzneimittels? Einen Todesfall?»

Eine Doppelbelastung hat ihn nicht interessiert, er wollte nicht mit einem Bein in der klinischen 
Praxis und mit dem anderen in der klinischen Forschung stehen. Und warum hat er das Büro dem Sprechzimmer vorgezogen? Hier zögert er, Ambivalenz wird spürbar: «Sobald man sich um Einzelschicksale kümmert, muss man jederzeit erreichbar sein.» Oder anders gesagt: Man verliert einen grossen Teil seiner Autonomie. Ein zentraler Begriff für Heiner Frost, nicht bloss auf sich selber bezogen. Auch auf die Patienten: «Niemand muss ein Medikament einnehmen, wenn er das nicht will. Übrigens: Ärzte sollten auch mal den Mut haben, nichts zu geben.»

Gleich wie er Autonomie für Individuen beansprucht, fordert er auch Souveränität für eine Behörde

\section{Auch vor dem Computer spürt ein Mediziner die Last der Verantwortung.}

wie Swissmedic. Ideen, wonach das Schweizerische Institut Kompetenzen an die EU abtreten könnte, hat er deshalb immer abgelehnt. "Wir sind nicht in der $\mathrm{EU}$, können dort nicht mitbestimmen. Und wir sind ein bedeutender Standort für die Pharmabranche. Deshalb wäre es das Falscheste, wenn wir in der Schweiz kein eigenes Kompetenz- und Wissenszentrum mehr hätten, das unabhängig entscheiden kann.»

Speziell an diesem Bekenntnis ist unter anderem, dass es von einem ehemals Deutschen stammt, der vor 15 Jahren Schweizer wurde.

Heiner Frost kam vor 40 Jahren als Assistenzarzt in die Schweiz, zusammen mit dem Internisten Walter Siegenthaler, der eine ganze Mannschaft von Bonn nach Zürich mitnahm. «Auf vieles war ich nicht vorbereitet», erinnert er sich, «zum Beispiel dass mein Salär in der Schweiz wesentlich geringer war als in Deutschland. Oder dass ich plötzlich Ausländer war.» Zwei Reminiszenzen aus jener Zeit sind ihm unvergesslich geblieben: «Eines Tages - ich war damals junger Familienvater - erschien bei mir am Unispital ein Kantonspolizist in Zivil, um mich ausführlich auszufragen. Ob ich Verbindungen zum Osten oder zur RAF habe, wollte er wissen. Und das Zweite: In meinem Ausländerausweis hatte es damals einen roten Zettel. Darauf stand, dass Unzucht mit Minderjährigen einen sofortigen Landesverweis zur Folge haben würde.»

\section{Welt der Wissenschaft}

Als «goldene Zeit» bezeichnet Frost seinen Lebensabschnitt am Institut für Immunologie in Basel in den Siebzigerjahren. "Aus einem sehr strikten klinischen Betrieb in Zürich kam ich damals in die freie Welt der Wissenschaft. Forscher aus der ganzen Welt hatten das Ziel, das Immunsystem zu verstehen. Drei Nobelpreisträger gingen aus diesem Institut hervor.» Heiner Frost betrachtet die Immunologie noch heute als Schlüsselbereich der Medizin: «Die Evolution des Menschen war und ist sicher massgeblich geprägt vom Funktionieren des Immunsystems. Gerade in den vergangenen 20 Jahren haben wir ja hautnah erlebt, was eine Attacke auf das Immunsystem bedeuten kann. Der Fortschritt in diesem Bereich war enorm im 20. Jahrhundert - aber einen Impfstoff, der uns gegen eine HIV-Infektion schützen würde, gibt es immer noch nicht.» Heisst also weiterhin: Der Mensch muss sich selber schützen. «Die Verantwortung des einzelnen für sich selbst ist letztlich der Kernpunkt», ist Frost überzeugt - womit er auf den Autonomie-Begriff zurückkommt, der ihm so wichtig ist.

Hätte Heiner Frost selber entscheiden können, wie und wo es für ihn jetzt weitergeht, er hätte gerne noch ein paar Jahre in seiner Funktion bei Swissmedic weitergearbeitet, «mit 69 bin ich noch voll einsatzfähig. Aber die gesetzlichen Vorgaben zwingen mich zum Rückzug. In Europa sind wir halt furchtbar fixiert auf das Geburtsalter, in den USA gibt es diese Beschränkung nicht. Bei der dortigen Heilmittelbehörde, der FDA, wurde letzthin einer mit 91 pensioniert.» So oder so, Pension muss ja nicht Abstinenz bedeuten: «Ich werde am Fortschritt der Forschung über das humane Immunsystem weiterhin partizipieren.»

Im Wohnzimmer der Familie Frost steht ein Klavier. Es lenkt die Diskussion auf das, was für Heiner Frost jetzt, nach rund 50 Jahren Tätigkeit als Mediziner, an Bedeutung gewinnt: Goethe lesen, Memoiren schreiben, und eben - Klavier spielen: «Für mich ist das die ideale Kombination von motorischer, mentaler und gestalterischer Aktivität.»

Der Wissenschaftler wird spürbar auch dann, wenn er über Kunst spricht.

\section{Die nächste «Begegnung mit ...»}

Am Ende jeden Monats stellt die Schweizerische Ärztezeitung eine Persönlichkeit vor, die sich im Gesundheitswesen engagiert. Im November schildert Daniel Lüthi seine Begegnung mit Paul Daverio, Spezialarzt für Plastische und Wiederherstellungs- sowie Mikro-Chirurgie. 